\title{
Advanced Lung Carcinoma
}

National Cancer Institute

\section{Source}

National Cancer Institute. Advanced Lung Carcinoma. NCI Thesaurus. Code C153203.

A lung carcinoma that has spread extensively to other anatomic sites or is no longer responding to treatment. 